\title{
HOMOLOGY OPERATIONS ON A NEW INFINITE LOOP SPACE
}

\author{
BURT TOTARO
}

\begin{abstract}
Boyer et al. [1] defined a new infinite loop space structure on the space $M_{0}=\prod_{n \geq 1} K(\mathrm{Z}, 2 n)$ such that the total Chern class map $B U \rightarrow M_{0}$ is an infinite loop map. This is a sort of Riemann-Roch theorem without denominators: for example, it implies Fulton-MacPherson's theorem that the Chern classes of the direct image of a vector bundle $E$ under a given finite covering map are determined by the rank and Chern classes of $E$. We compute the Dyer-Lashof operations on the homology of $M_{0}$. They provide a new explanation for Kochman's calculation of the operations on the homology of $B U$, and they suggest a possible characterization of the infinite loop structure on $M_{0}$.
\end{abstract}

The total Chern class of the direct sum of two vector bundles, $c(E \oplus F)$, is not the sum but rather the product of the total Chern classes: $c(E \oplus F)=$ $c(E) c(F)$. In other words, for a topological space $X$, the total Chern class map $c: \widetilde{K}^{0} X \rightarrow \prod_{n \geq 1} H^{2 n}(X ; \mathbf{Z})$ is not a homomorphism of abelian groups for the obvious additive group structure on the set $\prod_{n \geq 1} H^{2 n}(X ; \mathbf{Z})$, but only for a multiplicative abelian group structure on it; let $\widetilde{M}^{0} X=1+\prod_{n \geq 1} H^{2 n}(X ; \mathbf{Z})$ with the appropriate multiplicative group structure. Since the group $\widetilde{K}^{0} X$ is the 0 th term of a cohomology theory $k^{*} X$ (0-connective $K$-theory), it is natural to expect that $\widetilde{M}^{0} X$ is also the 0 th term of a cohomology theory $\widetilde{M}^{*} X$, with the property that the map $c: \tilde{k}^{0} X=\widetilde{K}^{0} X \rightarrow \widetilde{M}^{0} X$ extends to a map of cohomology theories $\tilde{k}^{*} X \rightarrow \widetilde{M}^{*} X$. Recently Boyer, Lawson, Lima-Filho, Mann, and Michelsohn [1] defined a cohomology theory $\widetilde{M}^{*}$ with this property.

If $M_{0}$ denotes the infinite loop space such that $\left[X, M_{0}\right] \cong \widetilde{M}^{0} X$, then $M_{0} \simeq \prod_{n \geq 1} K(\mathbf{Z}, 2 n)$ as a space, and we know the $H$-space structure on $M_{0}$. (It is not the usual $H$-space structure on a product of Eilenberg-Mac Lane spaces.) In this paper we continue the analysis of the infinite loop space $M_{0}$ by computing the Araki-Kudo-Dyer-Lashof operations on $H_{*}\left(M_{0} ; \mathrm{Z} / p\right)$.

The computation starts with Kochman's calculation of these operations on $H_{*} B U$ [4], but there is a pleasant side effect to our analysis. We only need Kochman's calculation in the lowest nontrivial dimension, and with that as input, the rich structure of $M_{0}$ (it is the multiplicative $E_{\infty}$ space which underlies one component of an $E_{\infty}$ ring space) allows us to find all the operations on $H_{*} M_{0}$ and hence on $H_{*} B U$. In particular, one could say that the existence of BLLMM's theory gives a conceptual explanation for the simplicity of Kochman's formulas.

Received by the editors August 20, 1993.

1991 Mathematics Subject Classification. Primary 55S12; Secondary 55P47.

This paper was supported by an NSF postdoctoral fellowship. 
We also briefly describe the homology operations on another infinite loop space structure on $\prod_{n \geq 1} K(\mathrm{Z}, 2 n)$, this one defined by Segal, and we conclude with a conjectural characterization of BLLMM's theory.

I would like to thank Paulo Lima-Filho and Ben Mann for many conversations on their work. Also, Peter May taught a helpful class.

\section{Dyer-LAShof operations on THE COHOMOLOGY OF $M_{0}$}

Let $(i, j)=(i+j) ! / i ! j !$ if $i, j \geq 0$, and $(i, j)=0$ otherwise.

Theorem. Let $M_{0}$ be the infinite loop space defined by Boyer et al. There is a homotopy equivalence $M_{0} \rightarrow \prod_{n \geq 1} K(\mathbf{Z}, n)$ which we will specify in the proof. Let $l_{0}=1 \in H^{0}\left(M_{0} ; \mathbf{Z} / p\right)$ and, for $n \geq 1$, let $l_{n} \in H^{2 n}\left(M_{0} ; \mathbf{Z} / p\right)$ be the obvious element (pulled back from the generator of $\left.H^{2 n}(K(\mathbf{Z}, 2 n) ; \mathbf{Z} / p) \cong \mathbf{Z} / p\right)$. The coalgebra map $H^{*} M_{0} \rightarrow H^{*} M_{0} \otimes H^{*} M_{0}$ sends $l_{n}$ to $\sum_{i=0}^{n} l_{k} \otimes l_{n-k}$, and the dual Dyer-Lashof operations act on $l_{n}$ by the formulas

$$
Q_{r} l_{r(p-1)+s}=(-1)^{r+s}(s-1, r-s) l_{s}
$$

for $p$ odd, and

$$
Q_{2 r} l_{r+s}=(s-1, r+s) l_{s}, \quad Q_{2 r+1} l_{r+s}=0
$$

for $p=2$. Since $H^{*}\left(M_{0} ; \mathbf{Z} / p\right)$ is generated by the elements $l_{n}$ as an algebra over the Steenrod algebra, these formulas determine the action of the Dyer-Lashof operations on all of $H^{*}\left(M_{0} ; \mathbf{Z} / p\right)$ (and hence on $H_{*}\left(M_{0} ; \mathbf{Z} / p\right)$ ), via the diagonal Cartan formula and the Nishida relations $[9, p .6]$.

\section{DYER-LASHOF OPERATIONS ON HOMOTOPY GROUPS}

For the rest of the paper, it will be convenient to interpret the word "space" as meaning "simplicial set." Of course, statements about simplicial sets imply statements about topological spaces, by passing from a topological space to its total singular complex, which is a simplicial set [7]. The advantage of simplicial sets is that it is easy to work with the free (simplicial) abelian group $\mathbf{Z} X$ on a simplicial set $X$, with the property that $\pi_{*} \mathbf{Z} X=H_{*}(X ; \mathbf{Z})$. One could work with the free topological abelian group on a topological space $X$ instead, as in Dold-Thom [3], but the details are messier.

The infinite loop space $M_{0}$ has a lot of structure which is useful both for making calculations and for stating the results. In May's terminology of $E_{\infty}$ ring spaces [10, p. 145], what BLLMM proved is that there is an $E_{\infty}$ ring space $N \simeq \prod_{n \geq 0} K(\mathbf{Z}, 2 n)$, which is an abelian group under addition, such that there is a map of $E_{\infty}$ spaces $B U \rightarrow N$, where $N$ is given its multiplicative $E_{\infty}$ structure; and as a map of spaces this is the total Chern class map. The $E_{\infty}$ space $M_{0}$ mentioned in the Theorem is the connected component of $1 \in N$, with the multiplicative $E_{\infty}$ structure on this component. (Note that only the connected infinite loop space $B U$ maps into $M_{0} \subset N$; the infinite loop space $\mathrm{Z} \times B U$ does not map into either $M_{0}$ or $N$. I believe there is a version of BLLMM's theory into which $\mathrm{Z} \times B U$ does map, however.)

Here is the definition of "an $E_{\infty}$ ring space which is an abelian group under addition," or, as we will prefer to say, a May algebra. Fix an $E_{\infty}$ operad $\mathscr{C}$. 
Then a $\mathbf{Z} \mathscr{C}$-space is a $\mathscr{C}$-space $X$ which is also a (simplicial) abelian group such that the map $\mathscr{E}(n) \times X^{n} \rightarrow X$ is multilinear in the $n$ copies of $X$. We will give a useful variant of this definition in Lemma 1 . For a fixed $E_{\infty}$ operad $\mathscr{C}$, the $\mathbf{Z} \mathscr{C}$-spaces form a category. We define a May algebra to be a $\mathbf{Z} \mathscr{C}$-space for some $E_{\infty}$ operad $\mathscr{C}$. This section is devoted to stating the properties of May algebras.

In what follows, homotopy groups are always based at 0 . Also, we define $\pi_{*}(X ; \mathbf{Z} / p)$ for a simplicial abelian group $X$ to be $\pi_{*}(X \otimes \mathbf{Z} / p)$; in order that this group should satisfy the universal coefficient theorem relating it to $\pi_{*} X$, we need some additional assumption on $X$, e.g., that $X$ is torsion-free.

Lemma 1. For any space $X$, let $\mathrm{Z} X$ denote the free abelian group on $X$. Let $\mathscr{C}$ be an $E_{\infty}$ operad. If $X$ is a $\mathscr{C}$-space, then $\mathbf{Z X}$ is a $\mathbf{Z} \mathscr{C}$-space; in particular, it is a $\mathscr{C}$-space under multiplication. In fact, a $\mathbf{Z} \mathscr{C}$-space $X$ is exactly a space $X$ which is both $a \mathscr{C}$-space and an abelian group, in such a way that the map of abelian groups $\mathbf{Z X} \rightarrow X$ is also a map of $\mathscr{C}$-spaces.

We can summarize Lemma 1 by saying that the functor $X \mapsto \mathbf{Z X}$ is a monad [6] in the category of $\mathscr{C}$-spaces. A $\mathbf{Z} \mathscr{C}$-space is just an algebra over this monad.

Lemma 2. Let $\mathscr{C}$ be an $E_{\infty}$ operad and let $X$ be a $\mathbf{Z} \mathscr{C}$-space. Then there is a ring structure on the additive groups $\pi_{*} X$ and on $\pi_{*}(X ; \mathbf{Z} / p)$, and there are linear operations

$$
Q^{r}: \pi_{i}(X ; \mathbf{Z} / p) \rightarrow \pi_{i+2 r(p-1)}(X ; \mathbf{Z} / p)
$$

[for $\left.p=2, Q^{r}: \pi_{i}(X ; \mathbf{Z} / 2) \rightarrow \pi_{i+r}(X ; \mathbf{Z} / 2)\right]$, which satisfy the following properties:

(1) The ring structure and the operations $Q^{r}$ are natural with respect to maps of $\mathbf{Z} \mathscr{C}$-spaces.

(2) $\pi_{*} X$ and $\pi_{*}(X ; \mathbf{Z} / p)$ are graded-commutative rings.

(3) $Q^{r} x=0$ if $2 r<\operatorname{dim}(x)$ [if $\left.r<\operatorname{dim}(x)\right], x \in \pi_{*}(x ; \mathbf{Z} / p)$.

(4) $Q^{r} x=x^{p}$ if $2 r=\operatorname{dim}(x)$ [if $\left.r=\operatorname{dim}(x)\right], x \in \pi_{*}(X ; \mathbf{Z} / p)$.

(5) $Q^{r} 1=0$ if $r>0$, where $1 \in \pi_{0}(X ; \mathbf{Z} / p)$ is the identity element of the ring $\pi_{*}(X ; \mathbf{Z} / p)$.

(6) The external and internal Cartan formulas hold:

$$
Q^{r}(x \otimes y)=\sum_{i+j=r} Q^{i} x \otimes Q^{j} y
$$

if $x \otimes y \in \pi_{*}(X \otimes Y ; \mathbf{Z} / p)$, and $X, Y$ are $\mathbf{Z} \mathscr{C}$-spaces; and

$$
Q^{r}(x y)=\sum_{i+j=r}\left(Q^{i} x\right)\left(Q^{j} y\right)
$$

if $x, y \in \pi_{*}(X ; \mathbf{Z} / p)$.

(7) The Adem relations hold: If $p \geq 2$ and $r>p s$, then

$$
Q^{r} Q^{s}=\sum_{i}(-1)^{r+i}(p i-r, r-(p-1) s-i-1) Q^{r+s-i} Q^{i}
$$

if $p>2, r \geq p s$, and $\beta: \pi_{*}(X ; \mathbf{Z} / p) \rightarrow \pi_{*-1}(X ; \mathbf{Z} / p)$ denotes the Bockstein, 
then

$$
\begin{aligned}
Q^{r} \beta Q^{s}= & \sum_{i}(-1)^{r+i}(p i-r, r-(p-1) s-i) \beta Q^{r+s-i} Q^{i} \\
& -\sum_{i}(-1)^{r+i}(p i-r-1, r-(p-1) s-i) Q^{r+s-i} \beta Q^{i} .
\end{aligned}
$$

For a fixed prime $p$, we define the Dyer-Lashof algebra $\mathscr{R}$ as the associative graded algebra generated by symbols $Q^{r}, \beta Q^{r}$ subject to the Adem relations from property (7), above, and the "excess relations" which follow from property (3), above. We abbreviate Lemma 2 by saying that for a May algebra $X$, $\pi_{*}(X ; \mathbf{Z} / p)$ is an $\mathscr{R}$-algebra.

Lemma 3. Let $X$ be an $E_{\infty}$ space. The Dyer-Lashof operations on $\pi_{*}(\mathbf{Z X} ; \mathbf{Z} / p)$ $=H_{*}(X ; \mathbf{Z} / p)$, as in Lemma 2, are the usual Dyer-Lashof operations on $H_{*}(X ; \mathbf{Z} / p)$.

Lemma 4. Let $X$ be a May algebra. Then $H_{*}(X ; \mathbf{Z} / p)$, as an algebra over the Dyer-Lashof algebra $\mathscr{R}$, can be computed from $\pi_{*}(X ; \mathbf{Z} / p)$ as an algebra over $\mathscr{R}$. (Note that the operations on $H_{*}(X ; \mathrm{Z} / p)$ depend only on the multiplicative $E_{\infty}$ structure of $X$, while those on $\pi_{*}(X ; \mathbf{Z} / p)$ are defined using both the additive and multiplicative structures of $X$.)

Proof of Lemma 1. Let $X$ be an $E_{\infty}$ space; that is, $X$ is a $\mathscr{C}$-space with respect to an $E_{\infty}$ operad $\mathscr{C}$. The $\mathscr{C}$-space structure is defined by maps $\mathscr{C}(n) \times X^{n} \rightarrow X$ satisfying certain properties. Then $\mathrm{ZX}$ is a simplicial abelian group, and also a $\mathscr{C}$-space, the $\mathscr{C}$-structure being given by

$$
\mathscr{C}(n) \times(\mathbf{Z} X)^{n} \hookrightarrow \mathbf{Z} \mathscr{C}(n) \otimes(\mathbf{Z} X)^{\otimes n}=\mathbf{Z}\left(\mathscr{C}(n) \times X^{n}\right) \rightarrow \mathbf{Z} X .
$$

It is trivial to check that the functor $T: X \mapsto \mathrm{ZX}$ is a monad in the category of $\mathscr{C}$-spaces. This amounts to checking that the natural maps $X \rightarrow \mathbf{Z} X$ and $\mathbf{Z Z X} \rightarrow \mathbf{Z X}$, which are defined for any space $X$, are maps of $\mathscr{C}$-spaces if $X$ is a $\mathscr{C}$-space.

A monad $T$ defines a new category, the category of $T$-algebras. In this case, a $T$-algebra is precisely a $\mathscr{C}$-space which is also a simplicial abelian group, in such a way that the map $\mathbf{Z X} \rightarrow X$ preserves both structures. This is equivalent to the definition of a $\mathbf{Z} \mathscr{C}$-space at the beginning of this section. Q.E.D.

Proof of Lemma 2. Let $X$ be a May algebra. That is, there is an $E_{\infty}$ operad $\mathscr{C}$ such that $X$ is both a $\mathscr{C}$-space and a simplicial abelian group, and the $\Sigma_{n^{-}}$ equivariant map $\mathscr{C}(n) \times X^{n} \rightarrow X$ is multilinear in the $n$ copies of $X$. So this map extends to a $\Sigma_{n}$-equivariant map $\mathscr{C}(n) \times X^{\otimes n} \rightarrow X$ which is linear in $X^{\otimes n}$. Equivalently, we have a $\Sigma_{n}$-equivariant map of simplicial abelian groups, $\mathbf{Z} \mathscr{C}(n) \otimes X^{\otimes n} \rightarrow X$.

For a simplicial abelian group $E$, let $A(E)$ be the associated chain complex [7, p. 93], so that $H_{*}(A(E) ; \mathbf{Z})=\pi_{*} E$. In particular, $H_{*}(A(X) ; \mathbf{Z})=\pi_{*} X$. Then we have $\Sigma_{n}$-equivariant maps of chain complexes as follows:

$$
A(\mathbf{Z} \mathscr{C}(n)) \otimes A(X)^{\otimes n} \rightarrow A\left(\mathbf{Z} \mathscr{C}(n) \otimes X^{\otimes n}\right) \rightarrow A(X) .
$$

Here the first map is defined using the shuffle map $\eta: A(E) \otimes A(F) \rightarrow A(E \otimes F)$, which is a commutative and associative natural transformation, and the second 
map is the result of applying the functor $A$ to the map defined in the previous paragraph.

For each prime number $p$, the resulting map

$$
A(\mathbf{Z} \mathscr{C}(p)) \otimes A(X)^{\otimes p} \rightarrow A(X)
$$

makes the chain complex $A(X) \otimes \mathbf{Z} / p$ an object of the category $\mathscr{P}(p, \infty)$ of [8, pp. 160-161]. (The fact needed to check this is that the chain complex $A(\mathbf{Z} \mathscr{C}(p))=C_{*} \mathscr{C}(p)$ is a free $\Sigma_{p}$-resolution of $\mathbf{Z}$; see $[9, \mathbf{p} .7]$ for the details. Also, $A(X)$ satisfies the Cartan and Adem properties, by the arguments of $[9$, pp. 8-9].) This category is set up so that $H_{*}(A(X) \otimes \mathbf{Z} / p)=\pi_{*}(X ; \mathbf{Z} / p)$ is an algebra over the Dyer-Lashof algebra which satisfies the usual properties, as listed in Lemma 2. Q.E.D.

Proof of Lemma 3. This is automatic from the above construction of DyerLashof operations on homotopy groups. If $X$ is an $E_{\infty}$ space, then the Dyer-Lashof operations on $H_{*}(X ; \mathbf{Z} / p)$ are constructed in [9] by noting that the chain complex $C_{*}(X ; \mathbf{Z} / p)=A(\mathbf{Z} X) \otimes \mathbf{Z} / p$ is an object of the category $\mathscr{P}(p, \infty)$ mentioned above. Q.E.D.

Proof of Lemma 4. We observe that $X \mapsto \mathbf{Z} X$ is also a monad in the category of spaces, the corresponding algebras being the simplicial abelian groups. So, in particular, for a space $X$ there is an inclusion $X \rightarrow \mathbf{Z X}$, and for $X$ a simplicial abelian group, there is a retraction $\mathbf{Z} X \rightarrow X$. Thus, taking homotopy groups, we find that for any simplicial abelian group $X$, there is a natural surjection $H_{*}(X ; \mathbf{Z}) \rightarrow \pi_{*} X$ such that the composition $\pi_{*} X \rightarrow H_{*} X \rightarrow \pi_{*} X$ is the identity, where the first map is the Hurewicz map. Similarly, for $X$ a simplicial abelian group, there is a homomorphism $\mathbf{Z} / p X \rightarrow X \otimes \mathbf{Z} / p$, and so there is a natural surjection $H_{*}(X ; \mathbf{Z} / p) \rightarrow \pi_{*}(X ; \mathbf{Z} / p)$. And if $X$ is a May algebra, then Lemma 1 shows that $\mathbf{Z X} \rightarrow X$ is a map of May algebras, and Lemma 2 applied to this map shows that $H_{*}(X ; \mathbf{Z} / p) \rightarrow \pi_{*}(X ; \mathbf{Z} / p)$ is a map of algebras over the Dyer-Lashof algebra $\mathscr{R}$. (By Lemma $3, H_{*}(X ; \mathbf{Z} / p)$ is being given the structure of $\mathscr{R}$-algebra which it gets just from the multiplicative $E_{\infty}$ structure of $X$.)

The map $H_{*}(X ; \mathbf{Z} / p) \rightarrow \pi_{*}(X ; \mathbf{Z} / p)$ is defined for any simplicial abelian group $X$, so it is easy to describe explicitly. For example, if there is a map of simplicial abelian groups $X \rightarrow \prod_{n \geq 0} K(\mathbf{Z}, 2 n)$ which is a homotopy equivalence, then for $n \geq 1$ the map $H_{2 n}(X ; \mathbf{Z} / p) \rightarrow \pi_{2 n}(X ; \mathbf{Z} / p) \cong \mathbf{Z} / p$ is exactly the map $H_{2 n}(X ; \mathbf{Z} / p) \rightarrow H_{2 n}(K(\mathbf{Z}, 2 n) ; \mathbf{Z} / p) \cong \mathbf{Z} / p$, by the commuting diagram:

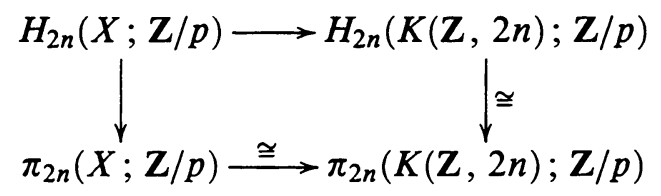

For $n=0$, the map $\bigoplus_{k \in \mathbf{Z}} \mathbf{Z} / p \cong H_{0}(X ; \mathbf{Z} / p) \rightarrow \pi_{0}(X ; \mathbf{Z} / p) \cong \mathbf{Z} / p$ sends the generator of $H_{0}$ of the $k$ th component of $X, k \in \mathbf{Z}$, to $k \in \mathbf{Z} / p$, since the composition $\pi_{0} X \rightarrow H_{0}(X ; \mathbf{Z} / p) \rightarrow \pi_{0}(X ; \mathbf{Z} / p)$ must be the natural map $\pi_{0} X \rightarrow \pi_{0}(X ; \mathbf{Z} / p)$. (The Hurewicz map $\pi_{0} X \rightarrow H_{0}(X ; \mathbf{Z} / p)$ is not a homomorphism of groups, but this argument works anyway.) 
(Warning. The multiplication and the Dyer-Lashof operations on $\pi_{*}(X ; \mathbf{Z} / p)$ are defined using the whole structure of $X$ as a May algebra. By contrast, the multiplication and the Dyer-Lashof operations on $H_{*}(X ; \mathbf{Z} / p)$ use only the multiplicative $E_{\infty}$ structure of $X$. One point which confused me for a while is that for a May algebra $X$, the $\bmod p$ Hurewicz map $\pi_{*}(X ; \mathbf{Z} / p) \rightarrow$ $H_{*}(X ; \mathbf{Z} / p)$, even if one can see how to define it, does not seem to preserve the Dyer-Lashof operations. The explanation is that the map $X \rightarrow \mathbf{Z} X$ which gives the Hurewicz map on homotopy groups preserves $X$ 's multiplicative infinite loop space structure but not the additive structure. The map $\mathbf{Z X} \rightarrow X$ preserves both structures.)

Thus we have a map $H_{*}(X ; \mathbf{Z} / p) \rightarrow \pi_{*}(X ; \mathbf{Z} / p)$ of algebras over the DyerLashof algebra $\mathscr{R}$. Dually speaking, we have a map

$$
\pi_{*}(X ; \mathbf{Z} / p)^{*} \rightarrow H^{*}(X ; \mathbf{Z} / p)
$$

of coalgebras over $\mathscr{R}^{\mathrm{op}}$, which is $\mathscr{R}$ with the opposite order of multiplication. But $H^{*}(X ; \mathbf{Z} / p)$ has additional structure: it is also an algebra over the Steenrod algebra. We have the usual formulas relating Steenrod operations and products to Dyer-Lashof operations and coproducts, as listed in $[9$, p. 6]. In fact, since $X$ is weakly homotopy equivalent to a product of Eilenberg-Mac Lane spaces as a simplicial abelian group, we can apply Serre's calculation of the cohomology of Eilenberg-Mac Lane spaces to see that $H^{*}(X ; \mathbf{Z} / p)$ is generated as an algebra over the Steenrod algebra by the image of $\pi_{*}(X ; \mathbf{Z} / p)^{*} \rightarrow H^{*}(X ; \mathbf{Z} / p)$. Consequently, if we know $\pi_{*}(X ; \mathbf{Z} / p)^{*}$ as a coalgebra over $\mathscr{R}^{\text {op }}$, then we know $H^{*}(X ; \mathbf{Z} / p)$ with the same structure. Q.E.D.

\section{Proof OF THE MAIN THEOREM}

In this section, we prove the theorem stated in $\S 1$, using Kochman's results heavily. In the next section we give a slightly longer proof, which has the advantage of only using Kochman's results in the lowest nontrivial dimension. As usual, all homotopy groups are based at 0 . Homotopy and homology groups will be understood to have $Z / p$ coefficients from now on. Also, I will assume $p \geq 3$, since the arguments for $p=2$ differ only notationally.

The idea of the proof is simple. Let $N$ be the May algebra discussed in $\S 2$. There are maps $\left(\pi_{*} N\right)^{*} \rightarrow H^{*} N \rightarrow H^{*} B U$ of coalgebras over $\mathscr{R}$ op such that the composition is injective. Since Kochman computed $H^{*} B U$ as a coalgebra over $\mathscr{R}^{\mathrm{op}}$, we can read off the corresponding structure for $\left(\pi_{*} N\right)^{*}$. By Lemma $4, H^{*} N$ is determined as a coalgebra over $\mathscr{R}^{\mathrm{op}}$ by $\left(\pi_{*} N\right)^{*}$, and this will complete the proof of the theorem.

Thus the calculation rests on two properties of BLLMM's theory: its relation to $K$-theory (giving the map $H^{*} N \rightarrow H^{*} B U$ ), and its May-algebra structure (giving the map $\left.\left(\pi_{*} N\right)^{*} \rightarrow H^{*} N\right)$. The latter is crucial, because the map $H^{*} N \rightarrow H^{*} B U$ by itself is not injective, so we cannot just read off the structure of $H^{*} N$ from that of $H^{*} B U$.

Let us begin the detailed proof. We know [1] that $N$ is an $E_{\infty}$ space with a compatible abelian group structure; that is, $N$ is a May algebra. We know that $N \simeq \prod_{n \geq 0} K(Z, 2 n)$. In fact, the abelian group structure of $N$, together with Lawson's choice of isomorphisms from the even-dimensional homotopy groups of $N$ to $\mathrm{Z}$, determines the map $N \rightarrow \prod_{n \geq 0} K(\mathrm{Z}, 2 n)$ uniquely, in 
the homotopy category of simplicial abelian groups (defined by inverting weak equivalences). (This follows from the universal coefficient theorem for Hom of chain complexes [2, Theorem VI.3.1a, p. 114].) Also, from the definition of $N$ it is clear that $N$ is torsion-free, so that the universal coefficient theorem allows us to compute $\pi_{*} N$ (by which we mean $\pi_{*}(N ; \mathbf{Z} / p)$ ) : $\pi_{2 n} N=\mathbf{Z} / p$ for $n \geq 0$, and $\pi_{2 n+1} N=0$.

We know that there is a map $B U \rightarrow N$ which is an $E_{\infty}$ map with respect to the multiplicative $E_{\infty}$ structure on $N$ [1]. ( $B U$ maps into the component of 1 , not 0 .) So we have maps $\left(\pi_{*} N\right)^{*} \rightarrow H^{*} N \rightarrow H^{*} B U$ of coalgebras over $\mathscr{R}$ op. If $\kappa_{n}$ denotes the generator of $\left(\pi_{2 n} N\right)^{*} \cong \mathbf{Z} / p$, we have $\kappa_{n} \mapsto l_{n} \mapsto c_{n}$, where $l_{n} \in H^{2 n} N$ denotes the $\bmod p$ reduction of the cohomology class which is represented by the map $N \simeq \prod_{r \geq 0} K(\mathbf{Z}, 2 r) \rightarrow K(\mathbf{Z}, 2 n)$, and $c_{n}$ denotes the $n$th Chern class. (The description of the map $\left(\pi_{*} N\right)^{*} \rightarrow H^{*} N$ follows from the proof of Lemma 4, and the description of the map $H^{*} N \rightarrow H^{*} B U$ is from [5].)

We see that the composition $\left(\pi_{*} N\right)^{*} \rightarrow H^{*} B U$ is injective, so we can read off the structure of $\left(\pi_{*} N\right)^{*}$ as a coalgebra over $\mathscr{R}^{\text {op }}$ from the known results for $H^{*} B U$. First for the coalgebra structure: since $\psi\left(c_{n}\right)=\sum_{i=0}^{n} c_{i} \otimes c_{n-i}$ in $H^{*} B U$ (this just amounts to the formula for the Chern classes of a direct sum of two vector bundles), we have $\psi\left(\kappa_{n}\right)=\sum_{i=0}^{n} \kappa_{i} \otimes \kappa_{n-i}$ in $\left(\pi_{*} N\right)^{*}$. It follows that $\pi_{*} N$ is a polynomial ring: $\pi_{*} N=\mathbf{Z} / p[u]$, $\operatorname{dim} u=2$. (With $\mathbf{Z}$ coefficients, we would have $\pi_{*} N=\mathrm{Z}[u], \operatorname{dim} u=2$.) It also follows that

$$
\psi\left(l_{n}\right)=\sum_{i=0}^{n} l_{i} \otimes l_{n-i}
$$

in $H^{*} N$. That is, we have computed the multiplicative $H$-space structure on $N$. (This was found by Lawson and Michelsohn with a different proof [5].)

Since $\left(\pi_{i} N\right)^{*}$ is nonzero only for $i$ even, and since it is 1-dimensional in that case, the Dyer-Lashof operations on $\left(\pi_{*} N\right)^{*}$ have the form

$$
Q_{r} \kappa_{r(p-1)+s}=a_{r s} \kappa_{s}
$$

$a_{r s} \in \mathbf{Z} / p$. (Here $Q_{r}:\left(\pi_{i+2 r(p-1)} X\right)^{*} \rightarrow\left(\pi_{i} X\right)^{*}$ denotes the dual map to $Q^{r}: \pi X \rightarrow \pi_{i+2 r(p-1)}$. Since dualizing reverses the order of multiplication, we should really write $Q_{r}$ to the right of its argument, e.g., " $x Q_{r}$.") Since the maps $\left(\pi_{*} N\right)^{*} \rightarrow H^{*} N \rightarrow H^{*} B U$ preserve these operations, we have

$$
Q_{r} l_{r(p-1)+s}=a_{r s} l_{s}, \quad Q_{r} c_{r(p-1)+s}=a_{r s} c_{s} .
$$

In particular, the existence of BLLMM's theory, together with its relation to $K$-theory, is enough to imply the general form of the Dyer-Lashof operations on $H^{*} B U$ : the basic operations send a Chern class to a multiple of a Chern class, not to some complicated polynomial in the Chern classes. This description of the operations on $H^{*} B U$ was discovered by Kochman [4]. In fact Kochman actually determined the numbers $a_{r s}: a_{r s}=(-1)^{r+s}(s-1, r-s)$, where $(i, j)=$ $(i+j) ! / i ! j !$ if $i \geq 0$ and $j \geq 0$, and $(i, j)=0$ otherwise.

We can then go back and use Kochman's calculation of the numbers $a_{r s}$ to determine the operations in $H^{*} N$ :

$$
Q_{r} l_{r(p-1)+s}=(-1)^{r+s}(s-1, r-s) l_{s} .
$$

Q.E.D. 


\section{ANOTHER PROOF OF THE MAIN THEOREM}

I will continue to assume that the prime $p$ is at least 3 , the case $p=2$ differing only trivially. We continue to use the notation of the previous section.

The point of this section is to compute the Dyer-Lashof operations on $\pi_{*} N$, and hence on $H^{*} N$ and $H^{*} B U$, using only Kochman's calculation that $Q_{2} c_{2 p-1}$ $=-c_{1}$ in $H^{*} B U$ (or, for $p=2$, that $Q_{4} c_{3}=c_{1}$ ). We will begin by computing $Q^{r} u$ in $\pi_{*} N$. The result is as follows:

Lemma 5. Let $N$ be the May algebra defined by Boyer et al. Then $\pi_{*}(N ; \mathbf{Z} / p)$ is the following algebra over the Dyer-Lashof algebra:

$$
\pi_{*}(N, \mathrm{Z} / p)=\mathbf{Z} / p[u],
$$

a polynomial ring on one generator $u$ of dimension 2 , with operations

$$
Q^{r} u=(-1)^{r+1} u^{r(p-1)+1}, \quad \beta Q^{r} u=0
$$

for $p$ odd (where $\beta: \pi_{*}(N ; \mathbf{Z} / p) \rightarrow \pi_{*-1}(N ; \mathbf{Z} / p)$ is the Bockstein operation), and $Q^{2 r} u=u^{r+1}, Q^{2 r+1} u=0$ for $p=2$.

Proof of Lemma 5. We take the computation that $\pi_{*}(N) \cong \mathbf{Z} / p[u]$ from the previous section. Since $\pi_{*}(N)$ is 0 in odd dimensions, it is trivial that $\beta Q^{r} u=$ 0 (or, for $p=2$, that $Q^{2 r+1} u=0$ ). Let $a_{r} \in \mathbf{Z} / p$ be the numbers such that $Q^{r} u=a_{r} u^{r(p-1)+1}$. In the previous section we used Kochman's calculations to show that $a_{r}=(-1)^{r+1}$. Here we will use only Kochman's result that $Q_{2} c_{2 p-1}=$ $-c_{1}$, i.e., that $a_{2}=-1$, and we will compute the other $a_{r}$ using the Adem relations, as in Lemma 2, part (7).

We want to exploit our knowledge that $a_{2}=-1$, or, in the form that we will use, $Q^{2} u=-u^{2 p-1}$. So it is natural to use the Adem relation for $Q^{r} Q^{2} u$, which says that, for $r \geq 2 p+1$,

$$
Q^{r} Q^{2} u=\sum_{i}(-1)^{r+i}(p i-r, r-2(p-1)-i-1) Q^{r+2-i} Q^{i} u .
$$

Since $u$ is only 2-dimensional, most of the terms in this sum turn out to be 0 . For the $i$ th term to be nonzero, $i$ must satisfy $p i-r \geq 0$, so that $i \geq r / p$. But also, since $Q^{i} u$ has dimension $2 i(p-1)+2$, Lemma 2, part (3) shows that the term $Q^{r+2} Q^{i} u$ can only be nonzero if

$$
2(r+2-i) \geq 2 i(p-1)+2,
$$

that is, $i \leq(r+1) / p$. Since $r / p \leq i \leq(r+1) / p$, there is at most one integer $i$ for which the $i$ th term in the above sum is nonzero; in fact, for $r \not \equiv 0,-1(\bmod p)$, the whole sum is 0 .

Thus the Adem relations in this situation take the form:

(a) $Q^{r} Q^{2} u=0$,

$$
\begin{array}{rlrlrl}
\text { (a) } \quad Q^{r} Q^{2} u & =0, & & r \not \equiv 0,-1(\bmod p), & r \geq 2 p+1 . \\
\text { (b) } Q^{p s} Q^{2} u & =Q^{(p-1) s+2} Q^{s} u, & r & =p s, & & r \geq 3 p . \\
\text { (c) } Q^{p s-1} Q^{2} u & =(s-1) Q^{(p-1) s+1} Q^{s} u, & r & =p s-1, & & r \geq 3 p-1 .
\end{array}
$$$$
\text { (b) } Q^{p s} Q^{2} u=Q^{(p-1) s+2} Q^{s} u \text {, }
$$

We now recall the notation $a_{r} \in \mathbf{Z} / p$ for the numbers such that $Q^{r} u=$ $a_{r} u^{r(p-1)+1}$. We can evaluate the right side of Adem relations (a), (b), (c), using 
Lemma 2 repeatedly. For (b) we have

$$
\begin{aligned}
Q^{(p-1) s+2} Q^{s} u & =Q^{(p-1) s+2}\left(a_{s} u^{s(p-1)+1}\right) \\
& =a_{s}(s(p-1)+1)\left(Q^{2} u\right)\left(Q^{1} u\right)^{s(p-1)} \\
& =(s-1) a_{s} u^{s p(p-1)+2 p-1},
\end{aligned}
$$

where we use $Q^{2} u=-u^{2 p-1}$. We can evaluate (c) similarly. To summarize the results:
(a) $Q^{r} Q^{2} u=0$, $r \not \equiv 0,-1(\bmod p), \quad r \geq 2 p+1$.
(b) $Q^{p s} Q^{2} u=(s-1) a_{s} u^{s p(p-1)+2 p-1}, \quad r=p s$, $r \geq 3 p$.
(c) $Q^{p s-1} Q^{2} u=(s-1) a_{s} u^{s p(p-1)+p}$
$r=p s-1$, $r \geq 3 p-1$.

By Lemma 2, part (4), we have $Q^{1} u=u^{p}$; so $a_{1}=1$. We use our knowledge that $Q^{2} u=-u^{2 p-1}$ to evaluate $Q^{r} Q^{2} u$, which is the left side of the Adem relations:

$$
\begin{aligned}
Q^{r} Q^{2} u & =-Q^{r}\left(u^{2 p-1}\right)=-\sum_{i+1+\cdots+i_{2 p-1}=r}\left(Q^{i_{1}} u\right) \cdots\left(Q^{i_{2 p-1}} u\right) \\
& =u^{(r+2)(p-1)+1}\left(a_{r-2 p+2}-\sum_{\substack{i_{1}+\cdots+i_{2 p-1}=r, \\
\text { all } i^{\prime} s<r-2 p+2}} a_{i_{1}} \cdots a_{i_{2 p-1}}\right) .
\end{aligned}
$$

Inspection of the above formulas for all $r \geq 2 p+1$ shows that all the numbers $a_{i}$ are determined by induction, given our knowledge that $a_{1}=1, a_{2}=-1$. (Note that it is not at all clear that this would be so with a different value of $a_{2}$ e.g., $a_{2}=0$.) We now check that $a_{r}=(-1)^{r+1}$ satisfies the equations, so this will be the unique solution.

Assuming that $a_{r}=(-1)^{r+1}$ (so as to check that the equations are verified), we evaluate the expression $Q\left(Q^{2} u\right)$ using the left side of the Adem relations. Note that only the terms $Q^{r} Q^{2} u$ with $r \geq 2 p+1$ will be relevant to the Adem relations.

$$
\begin{aligned}
Q\left(Q^{2} u\right) & =-Q\left(u^{2 p-1}\right)=-(Q u)^{2 p-1}=-\left(u^{p}-u^{2 p-1}+u^{3 p-2}-\cdots\right)^{2 p-1} \\
& =\left(\frac{u^{p}}{1+u^{p-1}}\right)^{2 p-1}=u^{p(2 p-1)} \sum_{k}(-1)^{k}(k, 2 p-2) u^{k(p-1)} \\
& =\sum_{r}(-1)^{r}(r-2 p+1,2 p-2) u^{2 p-1+r(p-1)} .
\end{aligned}
$$

We recall the usual formula for binomial coefficients $\bmod p$ :

Lemma $6([12$, p. 5]). Let $p$ be a prime number and $a, b$ nonnegative integers. Let $a=a_{0}+a_{1} p+\cdots, b=b_{0}+b_{1} p+\cdots$ be the $p$-adic expansions of $a$ and $b$. Then

$$
\left(\begin{array}{l}
a \\
b
\end{array}\right)=\left(\begin{array}{l}
a_{0} \\
b_{0}
\end{array}\right)\left(\begin{array}{l}
a_{1} \\
b_{1}
\end{array}\right) \cdots(\bmod p) .
$$

Assume that $r \geq 2 p+1$, which is necessary for the Adem relations we are considering. Then, in particular, $r-1 \geq 2 p-2$, so that $(r-2 p+1,2 p-2)=$ 
$\left(\begin{array}{c}r-1 \\ 2 p-2\end{array}\right)$. Now $2 p-2$ has $p$-adic expansion $(p-2)+(1) p$. Write $r-1=p t+i$, $0 \leq i<p$. Then, for $r \geq 2 p-1$,

$$
(r-2 p+1,2 p-2)=\left(\begin{array}{c}
r-1 \\
2 p-2
\end{array}\right)=\left(\begin{array}{l}
t \\
1
\end{array}\right)\left(\begin{array}{c}
i \\
p-2
\end{array}\right) \quad(\bmod p) .
$$

This is nonzero only if $i=p-2$ or $i=p-1$, that is, for $r \equiv 0$ or $-1(\bmod p)$. If $r \equiv 0(\bmod p)$, say $r=p s$, then

$$
\begin{aligned}
Q^{p s} Q^{2} u & =(-1)^{r}\left(\begin{array}{c}
s-1 \\
1
\end{array}\right)\left(\begin{array}{l}
p-1 \\
p-2
\end{array}\right) u^{2 p-1+r(p-1)} \\
& =(-1)^{s+1}(s-1) u^{2 p-1+p s(p-1)}
\end{aligned}
$$

where we have used the fact that $p$ is odd. Similarly, for $r=p s-1$, we have

$$
\begin{aligned}
Q^{p s-1} Q^{2} u & =(-1)^{p s-1}\left(\begin{array}{c}
s-1 \\
1
\end{array}\right)\left(\begin{array}{c}
p-2 \\
p-2
\end{array}\right) u^{2 p-1+(p s-1)(p-1)} \\
& =(-1)^{s+1}(s-1) u^{2 p-1+(p s-1)(p-1)}
\end{aligned}
$$

These agree with the right side of the Adem relations, as listed in (a), (b), (c) above, under our assumption that $a_{r}=(-1)^{r+1}$. Thus $a_{r}=(-1)^{r+1}$ satisfies the equations as claimed, and as explained above it is therefore the unique solution to these equations.

Thus we have proved that $a_{r}=(-1)^{r+1}$. Q.E.D. (Lemma 5)

We now finish our second proof of the main theorem. If $Q=\sum Q^{i}$ denotes the total Dyer-Lashof operation, Lemma 5 says that

$$
Q u=u^{p}-u^{2 p-1}+u^{3 p-2}-\cdots .
$$

We now work out the numbers $a_{r s}$; that is, we compute $Q\left(u^{s}\right)$, using the internal Cartan formula (Lemma 2, part (6)).

$$
\begin{aligned}
Q\left(u^{s}\right) & =(Q u)^{s}=\left(u^{p}-u^{2 p-1}+u^{3 p-2}-\cdots\right)^{s} \\
& =\left(\frac{u^{p}}{1+u^{p-1}}\right)^{s}=u^{p s} \sum_{k}(-1)^{k}(k, s-1) u^{k(p-1)} \\
& =\sum_{r}(-1)^{r+s}(r-s, s-1) u^{s+r(p-1)} .
\end{aligned}
$$

Thus $a_{r s}=(-1)^{r+s}(r-s, s-1)$. We have computed all the Dyer-Lashof operations on BLLMM's theory and on $K$-theory, starting from Kochman's calculation that $Q_{2} c_{2 p-1}=-c_{1}$.

\section{HoMOlogy OPERATIONS ON SEgal's INFINITE LOOP SPACE}

Segal earlier defined an infinite loop space structure on $\prod_{n \geq 1} K(\mathbf{Z}, 2 n)$ [11]. Segal's structure gives the same $H$-space structure on this space as BLLMM's coming from cup products in ordinary cohomology, but it did not have the desired property of making the Chern class map $B U \rightarrow \prod_{n \geq 1} K(\mathbf{Z}, 2 n)$ into an infinite loop map. Nonetheless, Segal's theory remains interesting; as the following calculations suggest, it seems to be the "simplest" infinite loop space with the given $H$-space structure.

As in BLLMM's theory, Segal makes $X=\prod_{n \geq 0} K(\mathbf{Z}, 2 n)$ into a May algebra. And as before, the problem reduces to computing the Dyer-Lashof operations on $\pi_{*} X=\mathbf{Z} / p[u], \operatorname{dim} u=2$. The result is the opposite extreme from 
the result for BLLMM's theory, where all the operations that could be nonzero were nonzero; for Segal's theory, we have, say for $p=2$ :

$$
Q^{2} u=u^{2}, \quad Q^{i} u=0, \quad i \neq 2 .
$$

Similarly for $p \geq 3$ :

$$
Q^{1} u=u^{p}, \quad Q^{i} u=0, \quad i \neq 1 .
$$

This follows easily from the fact that the map $H_{*} X \rightarrow \pi_{*} X$ preserves DyerLashof operations, and from the observation that in Segal's theory, the map

$$
\mathscr{C}(p) \times X^{p} \rightarrow X,
$$

which induces the Dyer-Lashof operations maps $\mathscr{C}(p) \times K(\mathbf{Z}, n)^{p}$ into $K(\mathbf{Z}, p n)$, so that $Q^{i} H_{*} K(\mathbf{Z}, n) \subset H_{*} K(\mathbf{Z}, p n)$.

As in the case of BLLMM's theory, we easily deduce the Dyer-Lashof operations on $H^{*} X$ :

$$
Q^{r}\left(l_{r(p-1)+s}\right)= \begin{cases}l_{s} & \text { if } r=s, \\ 0 & \text { otherwise. }\end{cases}
$$

\section{A QUESTION}

A May algebra is essentially an algebraic object. One can think of it as an $E_{\infty}$ object in the category of simplicial abelian groups.

One should be able to classify May algebras $X$ up to weak equivalence in some cases, e.g., if $\pi_{*} X$ is a polynomial ring $\mathbf{Z}[u], \operatorname{dim} u=2$. This includes both Segal's and BLLMM's theories. In particular, the calculations in this paper suggest that BLLMM's theory, localized at the prime 2 , should be the unique May algebra $N$ such that $\pi_{*} N=\mathbf{Z}_{(2)}[u]$ and $Q^{4} u=u^{3}$ in $\pi_{*}(N ; \mathbf{Z} / 2)$. Similarly, BLLMM's theory at an odd prime $p$ should be characterized by the properties $\pi_{*} N=\mathbf{Z}_{(p)}[u], Q^{2} u=-u^{2 p-1}$. Section 4 of this paper shows that these properties at least determine all the Dyer-Lashof operations on $\pi_{*} N$.

\section{REFERENCES}

1. C. P. Boyer, H. B. Lawson, Jr., P. Lima-Filho, B. M. Mann, and M.-L. Michelsohn, Algebraic cycles and infinite loop spaces, Invent. Math. 113 (1993), 373-388.

2. H. Cartan and S. Eilenberg, Homological algebra, Princeton Univ. Press, Princeton, NJ, 1956.

3. A. Dold and R. Thom, Quasifaserungen und unendliche symmetrische produkte, Ann. of Math. 67 (1958), 239-281.

4. S. O. Kochman, Homology of the classical groups over the Dyer-Lashof algebra, Trans. Amer. Math. Soc. 185 (1973), 83-136.

5. H. B. Lawson, Jr. and M.-L. Michelsohn, Algebraic cycles, Bott periodicity, and the Chern characteristic map, The Mathematical Heritage of Hermann Weyl, Proc. Sympos. Pure Math., vol. 48, Providence, RI, 1971, pp. 241-264.

6. S. Mac Lane, Categories for the working mathematician, Springer-Verlag, Berlin, 1971.

7. J. P. May, Simplicial objects in algebraic topology, Univ. of Chicago Press, Chicago, 1967.

8. __ A general algebraic approach to Steenrod operations, The Steenrod Algebra and its Applications, Lecture Notes in Math., vol. 168, Springer-Verlag, Berlin, 1970, pp. 153-171.

9. __ The homology of $E_{\infty}$ spaces, The Homology of Iterated Loop Spaces, Lecture Notes in Math., vol. 533, Springer-Verlag, Berlin, 1976, pp. 3-68. 
10. —_, $E_{\infty}$ ring spaces and $E_{\infty}$ ring spectra, Lecture Notes in Math., vol. 577, SpringerVerlag, Berlin, 1977.

11. G. Segal, The multiplicative group of classical cohomology, Quart. J. Math. Oxford 26 (1975), 289-293.

12. N. E. Steenrod and D. B. A. Epstein, Cohomology operations, Ann. of Math. Stud., no. 50, Princeton Univ. Press, Princeton, NJ, 1962.

Department of Mathematics, University of Chicago, 5734 S. University Avenue, CHICAGO, IlLINOIS 60637

E-mail address: totaro@math.uchicago.edu 